\title{
Plant Part
}

National Cancer Institute

\section{Source}

National Cancer Institute. Plant Part. NCI Thesaurus. Code C79611.

Any part of a plant that has not been processed further. 